\title{
Chemical Reactions in Solution: The New Photochemistry
}

\section{G. WILSE ROBINSON and WAYNE A. JALENAK}

\author{
Picosecond and Quantum Radiation Laboratory, Texas Tech University, \\ Lubbock, TX 79409, USA
}

Understanding the dynamics of chemical reactions in the condensed phase reaches a new plateau with each technological advance in time-resolved spectroscopy. Submicrosecond studies of the past revealed the role of long range molecular diffusion in condensed-phase chemistry and photochemistry. The picosecond $\left(10^{-12}-10^{-9} \mathrm{~s}\right)$ time scale, combined with the use of a high concentration of reactants, can provide new information about the "microdynamics" in the local region of the reaction itself. The role of solvent is particularly important: how it attaches to an activated reactant molecule, how it is displaced by the other reactant molecule preparatory to reaction, and how the solvent behavior affects the dynamics of single- and multi-channel processes, thus the relative yields of products in competing reactions. The theory presented here divides itself into two types: one that depends on a diffusion equation that also contains terms describing a distance-dependent reaction sink function and a reaction barrier; and a second type that deals phenomenologically with rate equations, including the rate of reactant/solvent interchange. Experiments subdivide naturally into steady state and transient measurements, the former dealing with quantum yields and steady state spectroscopic studies, the latter with picosecond transient spectroscopy. The two theoretical approaches can be interrelated in certain useful limits. The two types of experimental data, in combination with the theory, supply fundamental information about solvent participation in the local reaction region.

\section{PREAMBle}

On talking with various persons after my oral presentation, it became apparent to me that the intended message did not come across well in the twenty minutes allotted. We therefore have rewritten our paper in the hope of stating the message more clearly. We shall summarize some of the main points in this preamble. 
A chemical reaction in solution may be thought of as consisting of four steps, which of course are not always clearly separable: (1) relative diffusion of the reactants to a local reaction region, (2) microdynamics of solvent molecules and reactants in the local region, (3) the distance-dependent reaction, and (4) energy relaxation. The local region accommodates the reactants and attendant solvent molecules, and is characterized by energy barriers and wells ${ }^{1}$ that alter the course of the reaction. The barriers and wells are caused in one extreme by solvent molecules merely getting in the way, and in another by larger than normal intermolecular attraction between excited, charged, or otherwise activated reactants and neighboring solvent molecules. The local region encompasses a volume of about $10-50 \mathrm{~nm}^{3}$. Clearly, the dynamics of relative motion of the reactants in the local region differ from the dynamics outside this region. Emulating Emeis and Fehder, ${ }^{2}$ we denote the dynamics in this local region as the microdynamics of the reaction.

Parts (2) and (3) in the above scheme are the least understood. Yet they are the most important steps in determining the overall reaction dynamics under conditions of: high concentration of at least one of the reactants, high reaction barriers, or contributions from both of these sources under individually less extreme conditions. Only for extremely long-range reactive interactions do the microdynamics cease to play a role.

One can intuitively induce that steps (2) and (3) are also of utmost importance in determining the relative probabilities of $t w o$ or more channels for chemical reactions under equilibrium conditions in all solutions, dilute or concentrated. The question of solvent-dependent product yields in organic synthesis provides an important example.

While picosecond spectroscopy is perfectly capable of learning something about steps (2) and (3), it has not yet done so. Rather, emphasis has been placed mainly on step (4) as in the "caged" $I+I$ recombination problem, or on "molecular modeling" of the microdynamics without an adequate treatment of solvent participation. The main points of our paper are to indicate how information about steps (2) and (3) may be extracted from picosecond experiments, to guide in the selection of such experiments, and to show through two different theoretical analyses the meaning of certain parameters measured in these experiments. While the theories seem familiar, some important 
divergencies of thought are required for applications to picosecond experiments.

\section{INTRODUCTION}

A "new photochemistry" has arisen during the past few years because of improved instrumentation on the one hand and expanded curiosity on the other. The most notable improvements in the instrumentation concern first the light source, picosecond and subpicosecond pulse technologies ${ }^{3}$ are becoming commonplace; and second the creation of better defined microsystems through expansion-beam techniques. ${ }^{4}$ A number of presentations at this meeting have concerned these technologies. Improvements also in the ability to measure small fluorescence quantum yields have been made, but these are less striking.

Curiosity, fueled by these technological improvements, is being extended beyond gas-phase, isolated molecule phenomena into condensed phases. Though liquids and solids have formed the arena for many past photochemical studies, some crucial questions about the microdynamics of close-encounter reaction pairs (or groupings) have never before been seriously asked because of the absence of both the instrumentation and the theoretical framework by which to study such phenomena.

Since photochemistry has up to now been unable to deal with crucial questions about the microdynamics, it got itself into some very bad habits. These must be corrected for the purpose of dealing with the new photochemistry. For example, in solution photochemistry past emphasis was placed on diffusion controlled reactions. ${ }^{5}$ These tell us how close-encounter pairs come into being in the local reaction region through a relative diffusion process, but tell us almost nothing about the dynamics, once the close-encounter pairs are formed. We must therefore set aside much of what we have learned about diffusion controlled processes in favor of what has somewhat clumsily been termed the contra-diffusion-controlled limit. ${ }^{6}$ Our laboratory has also previously stressed ${ }^{7}$ that one must abandon the concept of featureless, infinitely fast reactions between the encounter pairs. The reactions are fast compared with the time constants that one typically measures 
in a diffusion controlled reaction, but clearly are not "infinitely fast." This particular bad habit includes, for example, the use of the "radiation" boundary condition in the Smoluchowski equation, on which much intuition about the dynamics and transient behavior of the encounter pairs has been based. ${ }^{8,9}$

In this paper we will concentrate on the simplest type of chemical reaction, the photochemical quenching reaction, where the lifetime of dilute excited molecules-probes-is perturbed by the presence of nearby molecular species-quenchers. Both probes and quenchers are dissolved in an "inert" solvent. This system illustrates the salient features of what we want to say without introducing the myriad of complexities that arise in reactions-such as $I+I$ recombinationwhere strong chemical bonds are broken or formed. Quenching reactions have an additional advantage: the decay of the probe in a fluorescence experiment directly provides the dynamics of interest. In the $I+I$ experiment, absorption measurements do not separate recombination from the energy relaxation step.

The outline of the paper is as follows. Two theoretical methods for treating this problem are introduced. The first method is a "concentration profile" method, where, in its simplest form, a Smoluchowski equation is solved for the concentration profile of quenchers around the probe. The Smoluchowski equation contains a distance-dependent "reaction sink term" together with a barrier placed in the local reaction region. This concentration profile, multiplied by the distancedependent reaction term, is integrated over all space to determine its contribution to the quenching dynamics. The second method is an ordinary kinetics method, with which is associated a fluorescence quantum yield formula. This formula is more general than equations published in the past by Weller, ${ }^{10}$ Eftink and Ghiron, ${ }^{11}$ and others, but reduces to these under various approximations.

Finally, we will show how the two theoretical methods are related to one another. This relationship indicates what parameters are actually being measured in a typical experiment. Any experiment must be designed to optimize the occurrence of the phenomena to be studied. This criterion sets the picosecond experiments apart from past experiments, since only at fairly high concentrations $(\geq 0.1 \%)$ of quencher does the ensemble contain a significant fraction of subsystems undergoing the microdynamical processes that one desires to measure. This work is therefore meant to interconnect the classical 
photochemistry, typically in extremely dilute solution, with the new photochemistry. It also interconnects steady state and dynamical measurements over this broad concentration range.

\section{CONCENTRATION PROFILE SCHEME}

One theoretical method that can be used to describe the microdynamics of a fluorescence quenching reaction is a concentration profile scheme, ${ }^{7}$ in which one solves a generalized diffusion equation to provide information about the distribution of quenchers around an average probe molecule. This distribution is dependent explicitly on the time following the production of the probes by excitation; and upon distances and angles, as well as the time derivatives of these variables. This computed distribution is then multiplied by the quenching reaction function, in general a function of all the foregoing variables except the time, and is integrated over all these variables to obtain the quenching contribution to the dynamics of the probe molecules. This scheme is modified if the distribution is distorted by memory effects, ${ }^{12}$ for example, but quenching can still be thought of as taking place from some effective distribution.

In this paper, some initial simplifications will be made in order to reduce the number of parameters required by the experimentalist; yet not so many approximations that the basic picture is lost. The approximations are as follows: (1) spherical symmetry, (2) well defined concentration profile at all relevant times, (3) full separation of the velocity and the space/time parts of the distribution at all relevant times, (4) velocity-independent quenching function, (5) a distanceindependent diffusion coefficient, and (6) a quenching rate that is proportional to the density of quenchers. The factorization of the distribution function into a purely velocity part and a space/time part depends upon the decay of the velocity autocorrelation function within the time scales of interest, such that at all relevant points in time and space the same (Maxwellian) velocity distribution exists. This factorization causes the Fokker-Planck equation to reduce to a Smoluchowski equation. ${ }^{13}$ The velocity-independent quenching rate removes the necessity of averaging the quenching rate over the fixed velocity distribution. Under these conditions, the generalized diffusion 
equation for the quenchers becomes, ${ }^{1}$

$$
\frac{d q}{d t}=\mathscr{D}\left\{\left[\frac{\partial}{\partial r}+\frac{2}{r}\right]\left[\frac{\partial}{\partial r}+\beta \frac{d u}{d r}\right]\right\} q-W q
$$

where $\beta=\left(k_{B} T\right)^{-1}, q=q(r, t)$ is the density of quenching species at a distance $r$ from the probe molecule at time $t, \mathscr{D}$ is the diffusion coefficient, $u=u(r)$ is the intermolecular potential of mean force between the quencher and the probe, and $W=W(r)$ is the quenching reaction function. The coordinate origin of the equation is fixed at the position of the probe molecule.

The initial condition is the quencher radial distribution $g(r)$ around the ground state molecule in the quencher/solvent environment. During and following excitation, this $g(r)$ evolves into $g^{\dagger}(r)$, the radial distribution function for quenchers surrounding the excited state molecule (the probe). If this evolution time-excitation followed by relaxation-is sufficiently short, then $q(r, 0) \approx g^{\dagger}(r)$. Otherwise the evolution of $g^{\dagger}(r)$ becomes a part of the microdynamics. The functions $g(r), g^{\dagger}(r)$, and $q(r, t)$ will all be scaled with the fractional concentration $0.0 \leqslant[Q] \leqslant 1.0$ of quencher. Naturally, this is an approximation for real mixtures.

Eq. (1) reflects the fact that as time evolves, probes that happen to have a high quencher concentration nearby disappear early in time, leaving an ensemble of probes having a sparser concentration of quenchers around them. Thus, on the average, the concentration profile of quenchers around probe molecules behaves as a wave moving away from the coordinate origin with time. The presence of diffusion terms in Eq. (1) opposes the motion of the concentration wave and leads to a steady state profile at long times. With no diffusion, as in a rigid glass, steady state is never reached-the quencher wave simply moves away as $\exp (-W t)$. Since the quenchers themselves are not disappearing, the above picture breaks down at very high quencher concentrations.

Eq. (1) also says that for a small diffusion coefficient, steady state is only slowly reached. Conversely, with a sufficiently short ranged $W(r)$ and reasonably high value of $\mathscr{D}$, solutions to Eq. (1), particularly those beyond the effective quenching range of $W(r)$, reach steady state rapidly. ${ }^{14}$ Scaling $q(r, t)$ does not affect the attainment of steady state. All the interesting dynamics of the concentration wave itself 
are then limited to the local reaction region. However, this is only a necessary, not a sufficient, condition for achievement of the contradiffusion-controlled reaction limit. As $[Q]$ is lowered, relative diffusion from a distance becomes the bottle-neck in the reaction dynamics however fast steady state is reached. See Section 4.

To obtain the relative probe dynamics, one adds the spontaneous (radiative plus non-radiative) rate $k_{p}$ for decay of probes to the convolution of $q=q(r, t)$ with $W(r)$,

$$
\frac{d p}{d t}=-k_{P} p-p \int_{0}^{\infty} d^{3} r W q
$$

where $p=p(t)$ represents the probe concentration. The procedure for determining the probe dynamics is first to solve Eq. (1) for $q$. This $q$ is then inserted into Eq. (2), which is solved for $p$.

The above analysis closely follows that in Ref. 7 for the donoracceptor problem. Differences arise because the quencher normally is a stable species. Thus, Eq. (1) omits a term $-k_{Q} q$. Also, the second term of Eq. (2) has a minus sign instead of a plus sign, since quenchers destroy probes, while donors create them. Difficulties arise in the quenching problem at high concentrations of quencher $(\geqslant 10 \%)$ because of the probable breakdown of the assumed density-dependent quenching rate, and the invalidity of the concept of a concentration wave moving out from a probe near the pure quencher limit. At very high quencher concentration ( $\geqslant 90 \%)$, Eq. (1) can be turned around so that $q$ represents the solvent. In that case, $W(r)=0$ everywhere, there is no effect of quenching on the distribution, and quenching occurs from an equilibrium distribution of quenchers around the probe. In the case of intermediate concentrations or when criteria for applicability of the Smoluchowski equation are not met, Eq. (1) is a poor way of obtaining $q$. Nonetheless, the validity of Eq. (2), albeit with an impenetrable $q$ function, permits correspondence with a kinetics scheme [see Section 6].

Any attempt to mimic a quenching reaction using Eqs. (1) and (2), must be accompanied by statements concerning (1) the initial condition, (2) the quenching function $W$, (3) the potential energy function $u$, and (4) the values of $\mathscr{D}$ and $k_{p}$. Eq. (1) can then be solved numerically. Figure 1 shows an example of $q$ for the following input parameters: $\mathscr{D}=4 \cdot 10^{7} \mathrm{~nm}^{2} / \mathrm{s}$; and a Gaussian barrier with $u(\max )=$ 


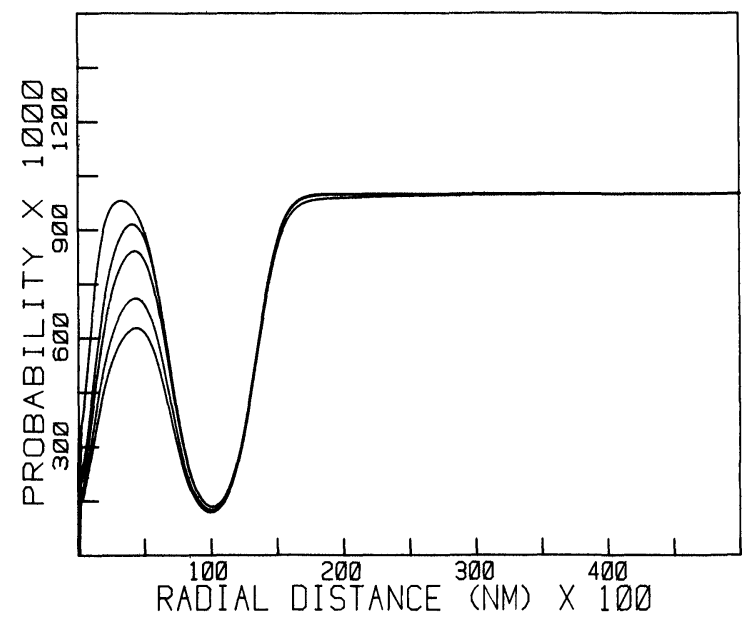

FIGURE 1 An example of $q(r, t)$ from Eq. (1). The input parameters are given in the text. The probability values given on the ordinate must be multiplied by the quencher concentration $0.0 \leqslant[Q] \leqslant 0.1$. Times from top to bottom are, $100 \mathrm{ps}, 800 \mathrm{ps,}$ $2.5 \mathrm{~ns}, 10 \mathrm{~ns}$, and $\sim 40 \mathrm{~ns}$. The latter time very nearly gives the steady state value of the distribution.

$2 k T, r(\max ) \equiv r_{0}=1.0 \mathrm{~nm}$, and half-width $\sim 0.5 \mathrm{~nm}$. The quenching function $W(r)$ decreases exponentially to zero at $r_{0}$ with a maximum of $6 \cdot 10^{10} \mathrm{~s}^{-1}$ at $r=0$, where, for purposes of plotting, $r=0$ represents the relative distance of "closest contact" between the quencher and the probe. The initial condition is $q(r, 0)=g^{\dagger}(r)=[Q] \exp (-\beta u)$, with $q(\infty, t)=[Q]$. No extreme conditions have been used: the barrier is of modest height, and the diffusion coefficient corresponds to a system having reasonable viscosity $(\eta \approx 20 \mathrm{cP})$. Clearly, for sufficiently large $[Q]$, the reaction pictured in Figure 1 is very close to the contradiffusion-controlled limit-beyond the barrier, quencher dynamics are of little importance.

\section{KINETICS SCHEME}

The classical photochemistry dealt primarily with quantum yields, not dynamics. In Weller's classic paper, ${ }^{10}$ an expression was given for fluorescence quantum yields in the presence of quenchers. The 
Appendix of a paper by Eftink and Ghiron ${ }^{11}$ contains a derivation of the classical photochemical quenching equations and shows that the much quoted Weller expression is a special case of a more general result. For the purpose of comparison between the concentration profile and kinetics schemes in picosecond experiments, a method that can be extended to high concentrations of quenchers must be utilized. This scheme was presented in an earlier paper. ${ }^{6}$ The general photochemical fluorescence quantum yield expression given there is,

$$
\frac{\Phi_{0}}{\Phi}=\frac{1+k_{1} Y / k_{0} X}{1+k_{1}^{f} Y / k_{0}^{f} X}
$$

where $\Phi_{0}$ is the fluorescence quantum yield of an excited molecule $A^{*}$ in an inert solvent, and $\Phi$ is the fluorescence quantum yield of $A^{*}$ in the presence of a certain concentration [Q] of quencher; $Y$ is the steady state concentration of quenched species, and $X$ is the steady state concentration of unquenched species under timeindependent excitation.

The meaning of the rate parameters $k_{0}, k_{1}, k_{0}^{f}$ and $k_{1}^{f}$ is apparent from the time-dependent equations describing the concentrations of quenched $y(t)$ and unquenched $x(t)$ excited state species,

$$
\begin{aligned}
& d x / d t=s+k_{2}^{\prime} y-\left(k_{0}+k_{2}\right) x \\
& d y / d t=s^{\prime}+k_{2} x-\left(k_{1}+k_{2}^{\prime}\right) y
\end{aligned}
$$

which refer to the following sequence of photochemical reactions,

$$
\begin{aligned}
A+h \nu & =A^{*} & & {[s(t)] } \\
A \cdots Q+h \nu^{\prime} & =A^{*} \cdots Q & & {\left[s^{\prime}(t)\right] } \\
A^{*} & =A & & {\left[k_{0}\right] } \\
A^{*} \cdots Q & =A \cdots Q & & {\left[k_{1}\right] } \\
A^{*}+Q & =A^{*} \cdots Q & & {\left[k_{2}, k_{2}^{\prime}\right] }
\end{aligned}
$$

where in Eq. (10) $k_{2}$ represents the forward reaction and $k_{2}^{\prime}$ the reverse reaction. Note that $k_{0}$ and $k_{1}$ are the total rate parameters for the spontaneous disappearance of $A^{*}$ and $A^{*} \ldots Q$, respectively. They contain the radiative $(f)$ as well as all nonradiative $\left(m_{i}, n_{j}\right)$ contributions:

$$
k_{0}=k_{0}^{f}+k_{0}^{m_{1}}+k_{0}^{m_{2}}+\cdots \quad \text { and } \quad k_{1}^{f}+k_{1}^{n_{1}}+k_{1}^{n_{2}}+\cdots
$$


The quantities $s(t)$ and $s^{\prime}(t)$ are time-dependent production rates of $A^{*}$ and $A^{*} \cdots Q$, respectively, by the light source. They depend on the light source intensity distributions across the absorption bands of $A$ and $A \cdots Q$, the absorption coefficients across these bands, and the temporal properties of the light source. When the evolution of $g(r)$ to $g^{\dagger}(r)$ is sufficiently short-lived compared with the inverse rate constants of interest, as is common even in picosecond experiments, the production rates can be ignored, since they don't sensibly affect the dynamics at $t>0$. The Eqs. (6)-(10) are an oversimplification in the sense that only a single quenched species $A^{*} \ldots Q$ is considered. If more than one mathematically distinguishable species is important, for example $A^{*} \cdots Q_{2}$ in addition to $A^{*} \cdots Q$, with different rate parameters, the analysis is more difficult. However, the simpler picture is favored by steric and symmetry factors, as well as the fact that, for reasonably large $k_{1}$ and quencher concentrations $\leqslant 10 \%, q$ is small in the region where reaction is actually taking place.

The fluorescence quantum efficiency is defined by,

$$
\Phi=\frac{\sum_{i} k_{i}^{f} X_{i}}{\sum_{i} k_{i} X_{i}}
$$

Usually, the relative efficiency, $\Phi_{0} / \Phi$, of Eq. (3) is employed. Summing Eq. (11) over the species for $\Phi_{0}$ and $\Phi$ yields Eq. (3).

To utilize Eq. (3), one must determine the ratio $Y / X$ in terms of the rate constants. The rate equations (4) and (5) are equally valid for the time-independent illumination used in the study of quantum yields. The steady state equations are,

$$
\begin{array}{r}
S+k_{2}^{\prime} Y-\left(k_{0}+k_{2}\right) X=0 \\
S^{\prime}+k_{2} X-\left(k_{1}+k_{2}^{\prime}\right) Y=0
\end{array}
$$

were $S$ and $S^{\prime}$ are constant production rates. These equations yield,

$$
\begin{gathered}
X=D^{-1}\left[\left(k_{1}+k_{2}^{\prime}\right) S+k_{2}^{\prime} S^{\prime}\right] \\
Y=D^{-1}\left[\left(k_{0}+k_{2}\right) S^{\prime}+k_{2} S\right]
\end{gathered}
$$

where

$$
D=k_{1}\left(k_{0}+k_{2}\right)+k_{0} k_{2}^{\prime}
$$


If $\alpha=S^{\prime} /\left(S+S^{\prime}\right)$ is the fractional production rate of the quenched species, then,

$$
\frac{Y}{X}=\frac{k_{2}+k_{0} \alpha}{\left(k_{1}+k_{2}^{\prime}\right)-k_{1} \alpha}
$$

This is the result given in Ref. 6. Combining Eq. (3) with Eq. (17) provides relative quantum yields in terms of the four rate parameters of Eqs. (8)-(10), the two purely radiative rate parameters, $k_{0}^{f}$ and $k_{1}^{f}$, and the quantity $\alpha$,

$$
\frac{\Phi_{0}}{\Phi}=1+\frac{k_{1}}{k_{0}}\left[\frac{k_{2}+k_{0} \alpha}{\left(k_{1}+k_{2}^{\prime}\right)-k_{1} \alpha}\right] / 1+\frac{k_{1}^{f}}{k_{0}^{f}}\left[\frac{k_{2}+k_{0} \alpha}{\left(k_{1}+k_{2}^{\prime}\right)-k_{1} \alpha}\right]
$$

Of interest is the special case considered by Eftink and Ghiron. ${ }^{11}$ Rewriting their most general formula [Eq. $\left(4^{\prime}\right)$ in their Appendix] in our notation gives,

$$
\frac{\Phi_{0}}{\Phi}=1+\frac{k_{i} k_{2} / k_{0}}{k_{1}+k_{2}^{\prime}+k_{2}\left(k_{1}-k_{i}\right) / k_{0}} / \frac{k_{1}+k_{2}^{\prime}+k_{2} k_{1}^{f} / k_{0}^{f}}{k_{1}+k_{2}^{\prime}+k_{2}\left(k_{1}-k_{i}\right) / k_{0}}
$$

A difference between all previous formulations and the present one is the occurance of the rate parameter $k_{i}$, which relates to "deactivation of the probe caused by the presence of the quenchers." In our formalism $k_{i}$ is not an independent quantity, but instead is absorbed as part of $k_{1}$ [ $\left(\tau_{q}^{-1}+k_{i}\right)$ of Ref. 11 equals $k_{1}$ of the present paper]. The parameter $k_{i}$ cannot in fact be separately evaluated without independent experimentation on the yields of remaining nonradiative processes.

Another difference lies in the interpretation of $k_{2}$ and $k_{2}^{\prime}$. In previous work, these rate parameters represent long range diffusion, $A^{*}$ relative to $Q$. However, at high [Q], this relative diffusion gives way to the microdynamics of the problem. The microdynamics are, at least in part, an extension into the local region of diffusional processes. This means that the magnitudes of $k_{2}$ and $k_{2}^{\prime}$ are expected to rise and fall with the value of the diffusion coefficient. However, features such as the height of barriers and their effect on the reaction dynamics are unknown quantities, which are not simply associated with the physical parameters normally describing diffusion. ${ }^{1,2,15}$ Thus, the microdynamics become a separate and unique part of the overall reaction process. This is a major part of the philosophy behind carrying out these studies. 
Multiplication of the numerator and denominator of Eq. (19) by $\left[k_{1}+k_{2}^{\prime}+k_{2}\left(k_{1}-k_{i}\right) / k_{0}\right]$ and collection of terms eliminates $k_{i}$ from the equation, showing that it indeed is not an independent quantity. Further multiplication of the numerator and denominator of the resulting expression by $\left(k_{1}+k_{2}^{\prime}\right)^{-1}$ yields,

$$
\frac{\Phi_{0}}{\Phi}=\frac{1+k_{1} k_{2} / k_{0}\left(k_{1}+k_{2}^{\prime}\right)}{1+k_{1}^{f} k_{2} / k_{0}^{f}\left(k_{1}+k_{2}^{\prime}\right)}
$$

which is wholly equivalent to the most general expression of Eftink and Ghiron. ${ }^{11}$ Comparing Eq. (20) with Eq. (18) shows therefore that the Eftink and Ghiron expression is a special case of our equation with $\alpha$ set equal to zero. Eftink and Ghiron further show that Weller's original result ${ }^{10}$ can be obtained from their most general equation by ignoring all nonradiative contributions except $k_{i}$. Thus, Weller's equation is also a special case of our Eq. (18).

According to our earlier paper, ${ }^{6}$ the solutions to the kinetics equations (4) and (5) are,

$$
\begin{aligned}
& x(t)=\frac{p(0)}{\lambda_{2}-\lambda_{1}}\left[K_{1} F_{2} e^{-\lambda_{1} t}-K_{2} F_{1} e^{-\lambda_{2} t}\right] \\
& y(t)=\frac{p(0)}{\lambda_{2}-\lambda_{1}}\left[K_{1} e^{-\lambda_{1} t}-K_{2} e^{-\lambda_{2} t}\right]
\end{aligned}
$$

where $(i=1,2)$,

$$
\begin{aligned}
2 \lambda_{i} & =\left(k_{0}+k_{1}+k_{2}+k_{2}^{\prime}\right)+(-1)^{i}(1+4 \gamma)^{1 / 2}\left(k_{1}-k_{0}+k_{2}^{\prime}-k_{2}\right) \\
K_{i} & =k_{2}+\left(k_{0}-\lambda_{i}\right) \alpha \\
F_{i} & =\left(\lambda_{i}-k_{0}-k_{2}\right) / k_{2} \\
\gamma & =k_{2} k_{2}^{\prime} /\left(k_{1}-k_{0}+k_{2}^{\prime}-k_{2}\right)^{2}
\end{aligned}
$$

$p(0)=s(0)+s^{\prime}(0)$ is the total concentration, $A^{*}+A^{*} \cdots Q$, at $t=0$ of the unquenched and the quenched probes; and $\alpha=s^{\prime}(0) / p(0)$ is the fraction of $p(0)$ that is $A^{*} \cdots Q$. The solutions are valid for picosecond pulse excitation, where $\Delta t$ (pulse) $\ll \lambda_{1}^{-1}, \lambda_{2}^{-1}$. These solutions can be verified by reinserting them into Eqs. (4) and (5), ignoring the production rates $s(t)$ and $s^{\prime}(t)$, and carrying out considerable algebra. 


\section{STATIC QUENCHING}

The presence of static quenching, which in the words of Weller ${ }^{10}$ concerns "directly excited $A \cdots Q$ complexes," seems to have been a source of confusion in the literature. Weller's Eq. (7) inclusive of static quenching has been used by many authors ${ }^{9}$ in an attempt to analyze deviations from Stern-Volmer behavior. ${ }^{16}$ We have seen, however, that Weller's formula depends on a number of approproximations: $k_{0}=k_{0}^{f}, k_{1}=k_{1}^{f}+k_{i}, k_{2}=k_{d}[Q] \ll k_{0}$, and $\alpha \ll 1$. Just at the point where static quenching becomes important, the latter two approximations break down badly, so it is no wonder that difficulties are encountered.

The degree of static quenching is given by the value of $\alpha$. In fact, one can think of $\alpha$ as a "coefficient of static quenching." In any actual experiment, $\alpha$ may not be simply related to [Q]. It would therefore be worthwhile if $\alpha$ could be obtained independently by experimental means, and provisionally this is possible. One solves Eq. (17) for $\alpha$,

$$
\alpha=\frac{Z(1+\varepsilon)-k_{2}}{\left(Z+k_{0}\right)}
$$

where $Z=k_{1} Y / X$ and $\varepsilon=k_{2}^{\prime} / k_{1}$. The value of $Y / X$ is determined experimentally from the quantities $\Phi_{0} / \Phi, k_{1}, k_{0}, k_{1}^{f}$, and $k_{0}^{f}$ according to Eq. (3). See Ref. 6 Table I for an actual experimental example. Even though $k_{2}$ and $k_{2}^{\prime}$ cannot always be separately determined, various methods may be found for getting around this obstacle and arriving at the value of $\alpha$ as a function of [Q]. Certainly as [Q] $\rightarrow 0$, $\alpha \rightarrow 0$. However, at large [Q] it is not necessary that $\alpha \rightarrow 1$, since relaxation to a particular quencher/solvent configuration, one that is nonexistent in the ground state, may be part of the microdynamics that one is studying.

\section{THEORETICAL INTERRELATIONSHIPS}

In the concentration profile scheme, with no loss of generality one may write $q$ as a steady state part plus a transient part, ${ }^{17}$

$$
q(r, t)=j(r)+f(r, t)
$$


where $\lim (t \rightarrow \infty) f(r, t)=0$. Inserting this into Eq. (2) yields,

$$
d p / d t=-k_{P} p-p \mathscr{W}-p W^{\prime}
$$

where

$$
W=\int_{0}^{\infty} d^{3} r W(r) j(r) \text { and } W^{\prime}=\int_{0}^{\infty} d^{3} r W(r) f(r, t)
$$

The integral $\mathscr{W}^{\prime}(t)$ vanishes at long times and/or extremely high quencher concentrations, yielding the steady state solutions,

$$
p(t)=p(0) \exp \left[-\left(k_{P}+\mathscr{W}\right) t\right]
$$

The quantity $p(t)$ of the concentration profile scheme is the total probe concentration. It must therefore be equivalent to $x(t)+y(t)$ of the kinetic scheme. Adding $x(t)$ and $y(t)$ of Eqs. (21) and (22), taking for definiteness the case where $\lambda_{2}>\lambda_{1}$, going to the long-time limit, and comparing the exponentials shows that,

$$
\lambda_{1}=k_{P}+\mathscr{W}
$$

Also, $k_{P}=k_{0}$, since $\mathscr{W}$ and $k_{P}$ are independent. In certain commonly occurring limits $\lambda_{1} \approx k_{0}+k_{2}$, in which case, $k_{2} \approx W$. Eq. (27) provides a connection between the two theoretical schemes.

In the foregoing, not only was the discussion of barriers absent, but also the distinction between $x(t)$ and $y(t)$ was destroyed when the sum was taken. Obviously, separating $x(t)$ and $y(t)$ is valid only for a sufficiently short ranged $W(r)$ [falling off rapidly compared with $r^{-3}$ ], such that the integration of Eq. (2) can be cut off at some finite radial distance. We denote this distance $r_{0}$. It is then possible to split $q(r, t)$ into two parts, one part $0 \leqslant r \leqslant r_{0}$ corresponding to efficient quenching, the other part $r>r_{0}$ to inefficient quenching. [Remember from Figure 1 that the relative distance $r=0$ corresponds not to superposed molecular centers, but to a distance of "closest approach"].

If there is a barrier, a natural location for $r_{0}$ is the radial distance specifying the top of the barrier, since $q(r, t)$ has a minimum here. For short ranged $W(r)$, the preponderance of the quenching certainly occurs inside of $r_{0}$. Under the condition that the reaction rate is linearly dependent on quencher density, as in Eq. (1), the fraction $f_{y}$ of probes that are under the influence of quenchers at steady state 
is then simply equal to the integral,

$$
g\left(r_{0}\right)=\Omega^{-1} \int_{0}^{r_{0}} d^{3} r j(r)
$$

where $\Omega$ is the integral of Eq. (28) with the pure quencher $g^{\dagger}(r)$ substituted for $j(r)$. Since $j(r)$ is scaled by [Q], $f_{y}$ decreases linearly with $[Q]$. At low $[Q]$, nearly all of the probes per unit time are just waiting around for quenchers to enter the local region by the long range relative diffusion process, while an immeasurable few are actually undergoing the microdynamics that one wishes to study. This is the reason why high $[Q]$ values are required for the achievement of the contra-diffusion-controlled limit.

Splitting the probes into the two parts also leads to further relationships between the concentration profile and kinetics schemes. To see this, ignore the production rates, add Eqs. (4) and (5), and set $y(t)=g\left(r_{0}\right) p(t)$ and $x(t)=\left[1-g\left(r_{0}\right)\right] p(t)$. Comparing the resulting relationships with the steady state equation discussed at the beginning of this section again yields $k_{0}=k_{P}$, but also,

$$
\left(k_{1}-k_{0}\right)=\mathscr{W}\left(r_{0}\right) / g\left(r_{0}\right)
$$

where $\mathscr{W}\left(r_{0}\right)$ is analogous to the integral in Eq. (25) except that it contains the factor $\Omega^{-1}$. It is thus seen for short range interactions that the parameter $\left(k_{1}-k_{0}\right)$ in the kinetics scheme is equal to $W(r)$ spatially averaged over the concentration profile of quenchers in its range of importance. At sufficiently long times, Eqs. (21) and (22) indicate that $f_{y}=\left(1+F_{2}\right)^{-1}$, so one has the additional relationships,

$$
g\left(r_{0}\right)=k_{2} /\left(\lambda_{2}-k_{0}\right)
$$

and,

$$
\mathscr{W}\left(r_{0}\right)=k_{2}\left(k_{1}-k_{0}\right) /\left(\lambda_{2}-k_{0}\right)
$$

Using the definitions of $\lambda_{1}$ and $\lambda_{2}$ and a bit of algebra, Eq. (31) can be shown to be fully consistent with Eq. (27). Various limiting forms of these equations may be derived under suitable approximations. As [Q] decreases, $g\left(r_{0}\right)$ and $k_{2}$ decline in value, and ultimately $k_{2}$ becomes identified with the long range diffusion process.

These relationships help to correlate the various parameters of the kinetics scheme with certain integrals in the steady state concentration 
profile scheme. At sufficiently high $[Q]$ and within the Smoluchowski model used, the kinetic rate constants are therefore related to the barrier height and other parameters of the concentration profile scheme. However, it is to be emphasized that the kinetics methods of Section 4 require neither an extremely short ranged $W(r)$ nor a barrier. In fact, it is the same scheme that has been used in diffusioncontrolled kinetics when transient terms are ignored. ${ }^{5}$

\section{TRANSIENT BEHAVIOR}

The transient terms ${ }^{18}$ in chemical reaction theory have always been somewhat of a puzzle. One may easily be lulled into the idea that during the time when $q(r, t)$ is moving towards its steady state value (see Figure 1), the rate parameters themselves are changing with time. At face value, then, during this induction period, Eqs. (21) and (22) would not seem to be valid solutions to the kinetics equations. However, in the contra-diffusion controlled limit, where diffusion plays a role only in the microdynamics, there are no missing time constants in the treatment described here. Therefore, any transient behavior that affects the dynamics or quantum yields, given by our Eqs. (3), (21) and (22), should be dependent only on the timeindependent rate parameters already introduced. To see this one first writes, following the discussion in Section 6,

$$
p(t)=p^{\prime}(t) \exp \left[-\left(k_{P}+\mathscr{W}\right) t\right]
$$

Recalling Eq. (27), the sum of Eqs. (21) and (22) shows that,

$$
p^{\prime}=\frac{p(0)}{\lambda_{2}-\lambda_{1}}\left[K_{1}\left(1+F_{2}\right)-K_{2}\left(1+F_{1}\right) e^{-\left(\lambda_{1}-\lambda_{1}\right) t}\right]
$$

Inserting Eq. (32) into Eq. (25) provides an equation for $p^{\prime}=p^{\prime}(t)$,

$$
d p^{\prime} / d t=-p^{\prime} W^{\prime}
$$

Combining Eqs. (33) and (34) gives,

$$
\mathscr{W}^{\prime}(t)=\frac{K_{2}\left(1+F_{1}\right)\left(\lambda_{2}-\lambda_{1}\right) e^{-\left(\lambda_{2}-\lambda_{1}\right) t}}{K_{2}\left(1+F_{1}\right) e^{-\left(\lambda_{2}-\lambda_{1}\right) t}-K_{1}\left(1+F_{2}\right)}
$$


All the constants are those of Eqs. (21) and (22), so no new information is gained through a study of the transient effects. Expression (35) is valid with or without the $r_{0}$ cut-off. Thus, Eqs. (3), (21) and (22) include the effects of transient behavior in the contra-diffusioncontrolled limit. Some of the above conclusions were already implied by preliminary experiments reported earlier. ${ }^{6}$ Equation (35), by the way, has the expected functional behavior. For example, for the case of a barrier with $r_{0}$ cut-off, and $t \approx 0$ and $\alpha \approx 0, W^{\prime}\left(r_{0}, t\right)$ is negative, indicating a growth of quenching towards steady state inside the barrier region. Conversely, the normal physical situation corresponds to a non-zero value of $\alpha$, in which case Eq. (35) leads to an initial decline of quenching in this region.

\section{REMAINING QUESTIONS}

Though we have dealt only with spherically symmetric reactions at the Smoluchowski level, we believe that, as long as the distribution function idea is a sound one, this paper can be used as a foundation to study more general models of chemical reactions in the condensed phase. The paper also serves to relate in a quantatative way the concepts of classical photochemistry with the distribution function approach. To our knowledge, this has never been done inclusive of the microdynamical features described here. Remaining questions at this level concern (1) angular dependent reactions, including those involving membranes, surfaces, or large molecules, (2) the quencher concentration dependence in the intermediate regime $(0.1 \leqslant[Q] \leqslant 0.9)$, and (3) whether or not the velocity-dependent part of the distribution plays a significant role. Beyond this level are questions, some currently being asked, ${ }^{19}$ that concern the breakdown of the barrier/distributionfunction picture altogether. After all, one should be averaging over the dynamics, not computing the dynamics from averaged distributions.

\section{Acknowledgements}

This problem originally derived from some investigations of biological fluorescence probe molecules. That research was supported by the National Institutes of Health [GM23765] beginnning around 1978. Some of the early work itself was supported by 
the National Science Foundation [CHE77-21913] and the Robert A. Welch Foundation [K-099(e)], but this work had to be temporarily discontinued during a lengthy period of construction in our laboratories. More recently this research has been supported in part both by the Welch Foundation and the NIH. A shortage of funds, including travel funds, was partially overcome by a grant from the G. Wilse Robinson, Jr. (father of GWR) Trust. Without these funds, the journey to Egypt, and the ensuing participation in the Photochemistry and Photobiology Conference, would never have materialized. GWR therefore acknowledges with sincere thanks Elizabeth Joyce, Trust Officer of the Commerce Bank of Kansas City, for the role she has indirectly played in this work. $\mathrm{He}$ also wishes to acknowledge three scientific colleagues, Professors L. S. Forster and R. A. Marcus, and Sir George Porter, for various discussions and criticisms that led to a more readable and useful manuscript. Finally, the authors are grateful to Dr. Jamine Lee of our laboratory for reading over a "final version" of the manuscript and helping to find some overlooked typographical errors in the equations.

\section{References}

1. S. H. Northrup and J. T. Hynes, J. Chem. Phys. 71, 871 (1979).

2. C. A. Emeis and P. L. Fehder, J. Am. Chem. Soc. 92, 2246 (1970).

3. R. K. Jain, Proc. Soc. Photo-Opt. Instr. Engrs. 322, 2 (1982); see also articles by various authors in Picosecond Phenomena III, Chemical Physics Series No. 23, eds. K. B. Eisenthal et al. (Springer-Verlag, Berlin, 1982).

4. D. H. Levy, Ann. Rev. Phys. Chem. 31, 197 (1980).

5. J. B. Birks, Photophysics of Aromatic Molecules (Wiley-Interscience, New York, 1970).

6. G. W. Robinson, R. A. Auerbach and J. A. Synowiec, Chem. Phys. Letters, 82, 219 (1981). In the present paper, some changes in notation have been made: in particular, $k_{3}$ and $k_{1}$ of Ref. 6 have been changed in the present work to $k_{1}$ and $k_{0}$ respectively. See also, G. W. Robinson and R. A. Moore, Soc. Photo-Opt. Instr. Engrs. 322, 224 (1982).

7. R. A. Auerbach, G. W. Robinson and R. W. Zwanzig, J. Chem. Phys. 72, 3528 (1980).

8. R. M. Noyes, Progr. React. Kinet. 1, 129 (1961).

9. W. R. Ware and J. S. Novros, J. Phys. Chem. 70, 3246 (1966).

10. A. Weller, Progr. React. Kinet. 1, 187 (1961).

11. M. R. Eftink and C. A. Ghiron, Analyt. Biochem. 114, 199 (1981).

12. B. Bagchi and D. W. Oxtoby, J. Chem. Phys. 77, 1391 (1982).

13. S. Chandrasekhar, Rev. Mod. Phys. 15, 1 (1943); S. H. Northrup and J. T. Hynes, J. Chem. Phys. 68, 3203 (1978); S. Nordholm, J. Chem. Ed. 59, 187 (1982).

14. W. A. Jalenak, unpublished data.

15. B. Bagchi and D. W. Oxtoby, to be published.

16. E. J. Bowen and W. S. Metcalf, Proc. Roy. Soc. (London), A206, 437 (1951).

17. U. Gösele, Physica (Utrecht) B85, 317 (1977).

18. See J. C. Andre, M. Bouchy and W. R. Ware, Chem. Phys. 37, 119 (1979), and earlier papers by these authors, for another point of view concerning reactions outside the diffusion-controlled limit.

19. E. H. Rezayi and H. Suhl, Phys. Rev. B25, 2324 (1982). 